\title{
Changes in Bone Mineral Density of the Proximal Femur and Contralateral Knee after Total Knee Arthroplasty: A 4-Year Follow-Up of 38 Patients
}

\author{
Jaroma Antti ${ }^{1 *}$, Soininvaara Tarja ${ }^{2}$ and Kröger Heikki ${ }^{1}$ \\ ${ }^{1}$ Department of Orthopedics, Traumatology and Hand Surgery, Finland \\ ${ }^{2}$ University of Eastern Finland, Finland
}

\section{ISSN: 2576-8875}

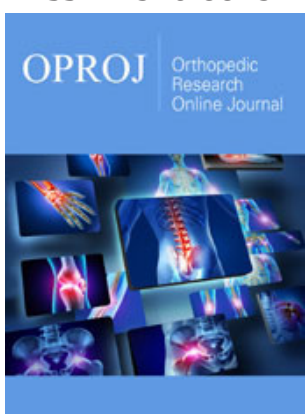

${ }^{* 1}$ Corresponding author:Jaroma Antti, Department of Orthopedics, Traumatology and Hand Surgery, Kuopio University Hospital, Kuopio, Finland

Submission: 侮 December 02, 2018 Published: 眥December 06, 2019

Volume 6 - Issue 2

How to cite this article: Jaroma A, Soininvaara T, Kröger H. Changes in Bone Mineral Density of the Proximal Femur and Contralateral Knee after Total Knee Arthroplasty: A 4-Year Follow-Up of 38 Patients. Ortho Res Online J. 6(2). OPROJ.000633.2019. DOI: $10.31031 /$ OPROJ.2019.06.000633

Copyright@: Jaroma Antti, This article is distributed under the terms of the Creative Commons Attribution 4.0 International License, which permits unrestricted use and redistribution provided that the original author and source are credited.

\begin{abstract}
Background: Previous studies have reported bone loss in the proximal femur and contralateral knee during the first year after total knee arthroplasty. We studied whether this bone loss continues or is amended.
\end{abstract}

Methods: Thirty-eight patients who were scheduled for unilateral total knee arthroplasty were examined by dual-energy X-ray absorptiometry. Measurements of the contralateral knee and both hips were performed within a week of the operation then 1,2 and 4 years postoperatively. The clinical status of the operated knee was recorded preoperatively and at each follow-up visit.

Results: The bone mineral density of the femoral neck and total femoral regions of interest of the affected side were significantly lower ( $6.6 \%$ and $5.1 \%$, respectively) than the values obtained for the contralateral hip, and these values remained unchanged throughout the 4-year follow-up period. In the contralateral hip, there was a significant decrease in femoral neck bone mineral density $(3.3 \%, \mathrm{p}<0.01)$ and total femoral bone mineral density $(3.0 \%, \mathrm{p}<0.001)$ at the 4 -year follow-up. In the contralateral knee, there was a significant reduction in bone mineral density of the femoral anterior metaphyseal $(5.0 \%, \mathrm{p}<0.001)$, total femoral metaphyseal $(3.6 \%, \mathrm{p}<0.001)$ and femoral diaphyseal $(5.1 \%, \mathrm{p}<0.001)$ regions of interest after 4 years when compared to baseline.

Conclusion: While total knee arthroplasty appears to stabilize affected side hip bone mineral density, it did not ameliorate the decrease in bone mineral density of the contralateral hip or contralateral distal femur during the 4 years after total knee arthroplasty.

Keywords: Total knee arthroplasty; Bone mineral density; Dual x-ray absorptiometry

Abbreviations: TKA: Total Knee Arthroplasty, OA: Osteoarthritis, BMD: Bone Mineral Density, DEXA: Dual X-ray Absorptiometry, BMI: Body Mass Index, AKS: American Knee Society Score, ROI: Region of Interest

\section{Introduction}

Total knee arthroplasty (TKA) provides pain relief and improves knee function in patients with osteoarthritis (OA) [1]. In theory, this functional improvement could also have a bone-preserving effect on the bone mineral density (BMD) of skeletal bones in TKA patients. However, several authors have raised concerns about the bone loss reported during the acute postoperative period after TKA in proximal femurs and the spine [2,3], as well as the contralateral knee [4]. Whether these changes are amended in the mid- to long-term is not clear due to a lack of BMD studies with a long enough follow-up period. The approximated rate of bone loss and the influencing factors are quite clear in an elderly population [5]. Furthermore, the mid-term periprosthetic bone changes after TKA have also been reported, with a clear decrease in BMD observed during the acute period, in addition to a change in the bone remodeling process due to stress shielding phenomenon, intraoperative trauma, postoperative immobilization in the recovery period and altered mechanical load [6,7]. Trabecular (metaphyseal) bone is considered to be the major site of bone remodeling [8]. The anterior flange of the femoral component protects the underlying periprosthetic metaphyseal bone against the shear forces of the extensor apparatus transmitted by patella and may be the explanation of the BMD decrease of the anterior femoral metaphyseal periprosthetic bone [6]. The diaphyseal BMD is considered to remain rather stable after the initial drop within 3-6 months [7]. 
Low BMD and aging are inevitably associated with increased fracture risk $[9,10]$. Some studies have also reported ipsilateral femoral neck fracture as a rare complication of TKA [3]. While there has also been evidence presented against proximal femoral BMD loss within a 2-year follow-up period after TKA, as evaluated by dual energy X-ray absorptiometry (DEXA) [11], concern remains due to more recent studies having shown the opposite results [2,3]. The aim of our study was to explore the mid-term changes in BMD, and to determine whether the BMD of the contra- and ipsilateral (affected side) hips and the contralateral (non-operated side) knee is preserved or even increased after TKA. We also focused on the possible correlations between BMD and functional status.

\section{Materials and Methods}

Thirty-eight patients who were scheduled to undergo unilateral TKA were recruited from the waiting list of the Orthopedic Department at Kuopio University Hospital between June 1997 and January 2000. Of these patients, $82 \%(n=31)$ were female. The mean age of the study population was 68 years (range $48-77$ years, SD 6.7 years) and the mean body mass index (BMI) was 29.8 (19.2-41.1, SD 5.2). Thirty-five of the patients had a knee with primary OA and two with posttraumatic OA (not including prior bone-affecting traumas,

Table 1: General baseline characteristics of 38 total knee arthroplasty patients followed up for 4 years.

\begin{tabular}{|c|c|}
\hline Gender: male/female & $7 / 31$ \\
\hline Operated side: right/left & $27 / 11$ \\
\hline Age (years) (min-max) (SD) & 68 (48-77) (6.7) \\
\hline Body mass index (min-max) (SD) & 29.8 (19.2-41.1) (5.2) \\
\hline Prosthesis model & 19 \\
Duracon modular & 13 \\
Anatomic Modular Knee (AMK) & 6 \\
\hline
\end{tabular}
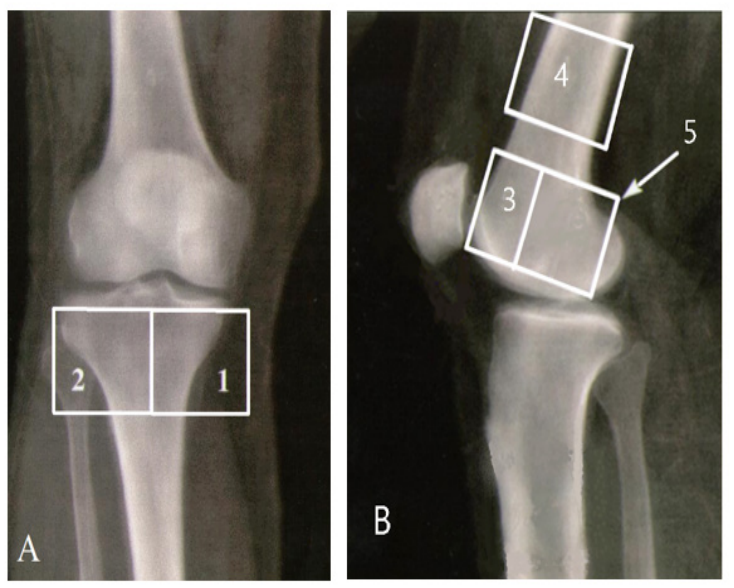

Figure 1: The regions of interest (ROIs) for bone mineral density (BMD) in the contralateral knee. Anteroposterior view (a) of medial metaphyseal (1) and lateral metaphyseal ROIs (2). Lateral view (b) of metaphyseal anterior (3), diaphyseal (4) and total metaphyseal ROIs (5) only open or arthroscopic meniscectomy). One patient had a bone necrosis of the lateral femoral condyle. Periprosthetic femoral and tibial BMD changes of the same study population were previously published [6,7]. Joint replacement of the contralateral knee or either hip before the scheduled surgery or during the follow-up period were considered exclusion criteria. Unilateral cemented TKA with all components cemented was performed on patients using standard techniques.

Patients were free from any diseases and were not taking any medications known to influence bone mineral metabolism, which was reconfirmed at every follow-up visit and BMD measurement appointment during the follow-up period. The female patients were all postmenopausal. A radiograph of the knee scheduled for operation was taken preoperatively. All patients were operated on by experienced orthopedic surgeons. Full weight-bearing was permitted immediately after the surgery. A continuous passive motion (CPM) device was used if necessary, to ensure early mobilization (12 patients, 31.6\%). The American Knee Society (AKS) score was used to clinically evaluate patient status and function of the operated knee during daily activities preoperatively and postoperatively at the follow-up visits at 1, 2 and 4 years. The baseline characteristics of patients and the prosthesis models used are presented in (Table 1). 
The BMD of the opposite knee and both hips was measured by fan-beam dual-energy X-ray absorptiometry (DEXA, Lunar Expert, Lunar Co., USA). A total of four measurements were taken, the first within a week of the operation and the others at 1,2 and 4 years postoperatively. In the contralateral knee, the regions of interest (ROIs) were both metaphyseal and diaphyseal to indicate the BMD changes of both the trabecular (metaphyseal) and the cortical (diaphyseal) bone (Figure 1). The femoral neck and total area were measured in the proximal femurs (Figure 2). Precision, expressed as the coefficient of variation (CV) for repeated BMD measurement of these ROIs, was $2.5 \%$ (range $2.2-2.9 \%$ ) in the proximal tibia and $3.2 \%(1.7-5.4 \%)$ in the distal femur in a study by Soininvaara [12]. For the proximal femur, the CV\% was $1.8 \%$ for the femoral neck and $0.9 \%$ for total proximal femur [13].

The study protocol was approved by the local ethics committee (decision number $71 / 97,13^{\text {th }}$ of May 1997). All patients gave written informed consent. The statistical analysis was performed using SPSS software, version 19 (SPSS Inc., Chicago, IL, USA). A mixed model based on linearly independent pairwise comparisons among the estimated marginal means was used to assess the correlation between measurements as well as to manage missing data. A mixed model was used to assess the association between the AKS score and changes in BMD at the measured ROIs at the four determined time points. In the model, the BMD measurements and the AKS scores were defined as random effects. Patient age at the time of the operation was tested as a covariant for BMD changes. The differences between the values of proximal femoral BMD of the contra-and ipsilateral hips were analyzed by paired-sample t-tests. A linear regression model was used to examine the correlation between BMD data and AKS and functional scores at all four time points. All analyzed BMD data were confirmed to be normally distributed by histograms of the residuals in the mixed model. The results are presented as the mean and standard error. P-values less than 0.05 were considered statistically significant.

\section{Results}

\section{Clinical evaluation and missing data}

The AKS score improved from the baseline mean value of 91 (SE 5.9) up to 170 (SE 6.0) in one year, and this improvement persisted until the end of the 4-year follow-up period (173 (SE 6.0) after 2 years and 164 (SE 6.1) after 4 years, $\mathrm{p}<0.001$ in all time points; (Table 2). Similar improvement was found for patient functional scores (Table 2). One AKS score and functional score was missing at the 1-and 2-year time points, and three scores were missing at the 4-year follow-up. All patients in the study visited the hospital for BMD measurements until 4-years postoperative. However, some femoral diaphyseal BMD data was lost (one patient throughout the follow-up, four individual measurements at the 1-year and two at the 2-year follow-up).

Table 2: Mean and standard error (SE) of American Knee Society (AKS) score, functional score and bone mineral density (BMD) of proximal femurs of 38 total knee arthroplasty patients over a 4-year follow-up.

\begin{tabular}{|c|c|c|c|c|}
\hline & Baseline & 1 year & 2 years & 4 years \\
\hline $\begin{array}{c}\text { AKS (SE) } \\
\text { Functional score (SE) }\end{array}$ & $\begin{array}{c}91(5.9) \\
55(4.0)(n=38)\end{array}$ & $\begin{array}{c}170(6.0)^{* * *} \\
85(4.0)^{* * *}(\mathrm{n}=37)\end{array}$ & $\begin{array}{c}173(6.0)^{* * *} \\
86(4.0)^{* * *}(n=37)\end{array}$ & $\begin{array}{c}164(6.1)^{* * * *} \\
80(4.0)^{* * *}(\mathrm{n}=35)\end{array}$ \\
\hline \multicolumn{5}{|c|}{ Region of interest (ROI) } \\
\hline $\begin{array}{c}\text { BMD neck } \\
\text { Affected } \\
\text { Contralateral }\end{array}$ & $\begin{array}{l}0.87(0.02)^{c} \\
0.92(0.02)\end{array}$ & $\begin{array}{l}0.85(0.02)^{c} \\
0.91(0.02)\end{array}$ & $\begin{array}{l}0.86(0.02)^{\mathrm{b}} \\
0.90(0.02)\end{array}$ & $\begin{array}{c}0.86(0.02)^{\mathrm{a}} \\
0.89(0.02)^{* *}\end{array}$ \\
\hline $\begin{array}{c}\text { BMD total } \\
\text { Affected } \\
\text { Contralateral }\end{array}$ & $\begin{array}{l}0.95(0.02)^{\mathrm{c}} \\
1.00(0.02)\end{array}$ & $\begin{array}{l}0.94(0.02)^{\mathrm{c}} \\
0.99(0.02)\end{array}$ & $\begin{array}{l}0.94(0.02)^{\mathrm{b}} \\
0.99(0.02)\end{array}$ & $\begin{array}{c}0.94(0.02)^{\mathrm{b}} \\
0.97(0.02)^{* * *}\end{array}$ \\
\hline
\end{tabular}

${ }^{* *} \mathrm{p}<0.01$ compared to baseline (mixed model)

$* * * \mathrm{p}<0.001$ compared to baseline (mixed model)

${ }^{a} \mathrm{p}<0.05$ between the affected and contralateral ROI BMD values (paired samples t-test, two-tailed significance) ${ }^{b} \mathrm{p}<0.01$ between the affected and contralateral ROI BMD values (paired samples t-test, two-tailed significance) ${ }^{c} \mathrm{p}<0.001$ between the affected and contralateral ROI BMD values (paired samples t-test, two-tailed significance)

\section{Proximal femoral bone mineral density}

The baseline postoperative BMD values of the affected side femoral neck and total femoral ROIs were significantly lower than the values of the contralateral side $(6.6 \%$ and $5.1 \%$, respectively; $\mathrm{p}<0.001$ ), and the difference remained significant throughout the follow-up period $(3.4 \%$ and $3.1 \%, \mathrm{p}<0.05$ and $\mathrm{p}<0.01$, respectively, at 4 years) (Table 2). For the hip on the affected side, there were no significant changes in BMD of the femoral neck or total femoral ROIs during the 4-year follow-up (Table 2). For the contralateral side hip, there was a significant decrease in femoral neck $(3.3 \%, \mathrm{p}<0.01)$ and total femoral $(3.0 \%, \mathrm{p}<0.001)$ BMD over 4 years (Table 2$)$. Patient 
age at the time of the operation was a significant covariate for both affected and contralateral side femoral neck BMDs ( $p=0.035$ and 0.015 , respectively) and total femoral BMD of the contralateral hip at baseline $(p=0.030)$. However, the age was not significantly associated with any BMD changes of the hips. The mean total BMD of the affected side proximal femur, despite being significantly lower than the contralateral side, was higher than age- and sex-matched public mean values, and the mean Z-scores were $+0.2 \mathrm{SD}$ to $+0.3 \mathrm{SD}$ during the follow-up period. Ten out of 38 patients (26.3\%) had Z-scores more than +1 SD, and this number increased to 15 out of 38 patients $(39.5 \%)$ at 4 years. On the other hand, out of the 38 patients, only one patient $(2.6 \%)$ at baseline and three patients at 4 years $(7.9 \%)$ were found to be osteoporotic according to the ethnic criteria as judged by a T-score below -2.5SD in the total measurement area.

\section{Changes in bone mineral density of the contralateral knee}

There was a significant BMD decrease in the anterior metaphyseal ROI at the 1-year follow-up (3.3\%, $\mathrm{p}=0.044)$, and this decrease continued up to 4 years postoperative $(5.0 \%, \mathrm{p}<0.001)$ (Table 3$)$. In the total femoral metaphyseal ROI, the decrease was also significant in the measurement performed after 1 year $(1.7 \%, \mathrm{p}=0.031)$ and 4 years $(3.6 \%, \mathrm{p}<0.001)$ compared to baseline (Table 3$)$. In the femoral diaphyseal ROI, there was a significant BMD decrease at the 2 -year $(2.9 \%, \mathrm{p}=0.036)$ and 4-year $(5.1 \%, \mathrm{p}<0.001)$ follow-up (Table 3). No statistically significant changes were found in the tibial metaphyseal measurements.

Table 3: Mean tibial and femoral bone mineral density values and standard error (SE) of the contralateral knee of 38 total knee arthroplasty patients over a 4-year follow-up period.

\begin{tabular}{|c|c|c|c|c|}
\hline Region of Interest (ROI) & Baseline & 1 year & 2 years & 4 years \\
\hline \multicolumn{5}{|l|}{ Tibial } \\
\hline Medial metaphyseal & $1.00(0.03)$ & $1.00(0.03)$ & $0.99(0.03)$ & $1.01(0.03)$ \\
\hline Lateral metaphyseal & $1.02(0.03)$ & $1.02(0.03)$ & $1.00(0.03)$ & $1.00(0.03)$ \\
\hline \multicolumn{5}{|l|}{ Femoral } \\
\hline Anterior metaphyseal & $1.20(0.03)$ & $1.16(0.03)^{*}$ & $1.16(0.03)^{*}$ & $1.14(0.03)^{*+*+}$ \\
\hline Total metaphyseal & $1.38(0.04)$ & $1.35(0.04)^{*}$ & $1.36(0.04)$ & $1.33(0.04)^{*+*+}$ \\
\hline Diaphyseal & $1.36(0.04)$ & $1.32(0.04)$ & $1.32(0.04)^{*}$ & $1.29(0.04)^{* * *, a}$ \\
\hline
\end{tabular}

${ }^{*} \mathrm{p}<0.05$ compared to baseline (mixed model)

$* * * \mathrm{p}<0.001$ compared to baseline (mixed model)

${ }^{\mathrm{a}} \mathrm{p}<0.05$ compared to the 1 -year measurement (mixed model)

\section{Correlations between bone mineral density and the} American Knee Society score and functional score

There was a positive correlation between the AKS score and BMD of the contralateral side hip total femoral ROI in the measurements taken 2 and 4 years postoperatively ( $r=0.414$ and $r=0.431$, respectively, $\mathrm{p}=0.011$ for both), as well as for the contralateral side femoral neck ROI at 4 years postoperatively $(r=0.411, p=0.016)$. The functional score was correlated with higher BMD values in the contralateral hip total femoral ROI at 1, 2 and 4 -years postoperative $(\mathrm{r}=0.374, \mathrm{r}=0.482$ and $\mathrm{r}=0.426, \mathrm{p}=0.023, \mathrm{p}=0.002$ and $\mathrm{p}=0.012$, respectively). In the measurements taken 4 years postoperative, the functional score also showed a positive correlation with the BMD of the contralateral femoral neck ROI ( $\mathrm{r}=0.391, \mathrm{p}=0.022)$.

\section{Discussion}

This mid-term follow-up study of 38 patients provided many interesting findings. Firstly, the contralateral hip BMD was higher at baseline and remained higher when compared to the side of the affected hip during the entire 4-year follow-up period. Similar, but non-significant results, were published by Kim et al. [3] in a study involving a preoperative assessment and a short postoperative period, as well as a 2-year follow-up study performed by Ishii et al. [11]. This difference began to level, evidenced by a significant decrease in BMD on the contralateral side, resembling age-related annual bone loss of $0.76 \%$ of femoral neck and $0.70 \%$ of total hip published by Greenspan et al. [14], but not on the hip of the affected side. Considering these results, we might expect that TKA could prevent further loss in BMD on the operative side, and even levelling the bone mineral balance between both hips. Patient age at the time of the operation was found to be significant covariate at the baseline, but it was not significantly associated with the BMD changes. We previously reported [4] that the baseline BMD of the proximal femur on the operative side was significantly lower than that of the contralateral side in all regions of interest, and this difference remained 1-year after TKA. In this study, we thought that the baseline BMD deficiency of the affected side represented disuse-based BMD loss as a result of preoperative exercise restriction due to pain and limiting daily living activities. 
The second major finding of the current study was that the distal femoral BMD of the control knee decreased during the midterm follow-up period. This decrease may be partly explained as a response of the metaphyseal bone to the immediate postoperative phase of impaired function. The minor change observed after the first postoperative year could again be considered an age-related phenomenon. Järvenpää et al. published distinctly deeper decrease in the same ROIs of periprosthetic BMD concerning mainly the same patient population $(16.8 \%$ in anterior metaphyseal, $20.1 \%$ in total metaphyseal and $11.1 \%$ in diaphyseal ROIs compared to $5.0 \%, 3.6 \%$ and $5.1 \%$ respectively in the contralateral knee in our study) [6]. This difference indicates the influence of stress shielding phenomenon of the femoral prosthesis component. Nevertheless, improved mobility after TKA could not ameliorate the preoperative disuse-associated bone loss in the distal femoral metaphysis and the $5.0 \%$ decrease of the anterior femoral metaphysis of the contralateral knee indicates, that stress shielding of the anterior flange of the femoral component is not entirely responsible for the BMD decrease of the anterior femoral metaphyseal periprosthetic bone. The tibial metaphysis BMD remained relatively stable, with no significant BMD losses or gains either in the early phase or during the follow-up. We could not identify any specific reason for this difference in metaphyseal bone behavior of the distal femur and proximal tibia.

Another finding was the positive correlation between better functional status of the patient and higher BMD values in the contralateral side hip, for which the decrease in BMD was statistically significant. Considering that this would help prevent BMD loss on the affected side, it might even further reduce the hip fracture risk. This is a promising result, as it suggests that a well-functioning knee prosthesis could also improve BMD. In the current study, however, we did not assess the radiological status of the hips. Therefore, we do not know the degree of arthrosis of these joints, which might influence both the BMD results and the functional capability of the subjects. On the other hand, study subjects who required total arthroplasty of either hips were excluded from the study, thus reducing the probability of considerable hip OA in the study population. As the measured hip ROIs are extra-articular, we might expect that sclerosis formation and the possible development of hip arthrosis to have a minimal effect on the BMD results. The strengths of our study are the long follow-up period, very few missing data and the study design, which is considered to be precise and reproducible. In order to determine whether the BMD difference between the affected and contralateral side hips equalized, we would need to perform a longer follow-up than the one undertaken in this study.

In summary, TKA could not improve mobility of the studied patient population enough to increase the BMD values of the hips or the contralateral knee. Nevertheless, it seemed to stabilize the BMD of the hip on the affected side, which was significantly lower than the contralateral side hip immediately after surgery. There was a positive correlation between good functional status and high BMD of the contralateral hip. However, we do not know if this was partly due to the development of arthrosis in these adjacent joints because the radiological status of these joints was not assessed. Nevertheless, a well-functioning total knee prosthesis may have capability to preserve BMD of the hips.

\section{Acknowledgement}

The authors thank research nurses Raija Kantanen, Eila Koski and Elina Jalava for technical assistance and biostatisticians Marja-Leena Lamidi and Tuomas Selander for assistance with statistics. The corresponding author thanks the State Research Fund of Finland and the Kuopio University Hospital Research Fund for financial help to conduct the study.

\section{Ethical Approval}

All procedures performed in studies involving human participants were in accordance with the ethical standards of the institutional and/or national research committee and with the 1964 Helsinki declaration and its later amendments or comparable ethical standards. The study protocol was approved by the local ethics committee (decision number 71/97, $13^{\text {th }}$ of May 1997). Informed consent was obtained from all individual participants included in the study.

\section{References}

1. Skou ST, Roos EM, Laursen MB, Rathleff MS, Arendt NL, et al. (2015) A randomized, controlled trial of total knee replacement. N Engl J Med 373(17): 1597-606.

2. Beaupre LA, Rezansoff A, Clark M, Jen H, Lambert RG, et al. (2015) Bone mineral density changes in the hip and spine of men and women 1-year after primary cemented total knee arthroplasty: Prospective cohort study. J Arthroplasty 30(12): 2185-2189.

3. Kim KK, Won YY, Heo YM, Lee DH, Yoon JY, et al. (2014) Changes in bone mineral density of both proximal femurs after total knee arthroplasty. Clin Orthop Surg 6(1): 43-48.

4. Soininvaara TA, Miettinen HJA, Jurvelin JS, Alhava EM, Kröger HPJ (2004) Bone mineral density in the proximal femur and contralateral knee after total knee arthroplasty. J Clin Densitom 7(4): 424-431.

5. Hannan MT, Felson DT, Dawson HB, Tucker KL, Cupples LA, et al. (2000) Risk factors for longitudinal bone loss in elderly men and women: The framingham osteoporosis study. J Bone Miner Res 15(4): 710-720.

6. Järvenpää J, Soininvaara T, Kettunen J, Miettinen H, Kröger H (2014) Changes in bone mineral density of the distal femur after total knee arthroplasty: A 7-year DEXA follow-up comparing results between obese and nonobese patients. Knee 21(1): 232-235.

7. Jaroma A, Soininvaara T, Kröger H (2016) Periprosthetic tibial bone mineral density changes after total knee arthroplasty. Acta Orthop 87(3): 268-273.

8. Feng X, McDonald JM (2011) Disorders of bone remodeling. Annu Rev Pathol 6: 121-145.

9. De Laet CE, Hout BA, Burger H, Hofman A, Pols HA (1997) Bone density and risk of hip fracture in men and women: Cross sectional analysis. BMJ 315(7102): 221-225

10. Cummings SR, Black DM, Nevitt MC, Browner W, Cauley J, et al. (1993) Bone density at various sites for prediction of hip fractures. The study of osteoporotic fractures research group. Lancet 341(8837): 72-75.

11. Ishii Y, Yagisawa K, Ikezawa Y (2000) Changes in bone mineral density of the proximal femur after total knee arthroplasty. J Arthroplasty 15(4): 519-522. 
12. Soininvaara T, Kröger H, Jurvelin JS, Miettinen H, Suomalainen O, et al. (2000) Measurement of bone density around total knee arthroplasty using fan-beam dual energy x-ray absorptiometry. Calcif Tissue Int 67(3): 267-272.

13. Huuskonen J, Väisänen SB, Kröger H, Jurvelin JS, Penttilä I, et al. (2002) Relation of sex hormones to bone mineral density in middle-aged men during a 4 year exercise intervention trial. Bone 31(1): 51-56.
14. Greenspan SL, Maitland LA, Myers ER, Krasnow MB, Kido TH (1994) Femoral bone loss progresses with age: A longitudinal study in women over age 65. J Bone Miner Res 9(12): 1959-1965.

For possible submissions Click below: 